$63^{\text {ème }}$ Congrès de la SFCO, 02006 (2015)

DOI:10.1051/sfco/20156302006

(C) Owned by the authors, published by EDP Sciences, 2015

\title{
COMMUNICATION
}

\section{Mise en place de canines mandibulaires incluses : un challenge chirurgico-orthodontique?}

\author{
Wagner $\mathrm{D}^{*, * *}$, Gros C-I**,***, Bolender $\mathrm{Y}^{*, * *}$, Bacon $\mathrm{W}^{*, * *}$, Bornert $\mathrm{F}^{* * * * * * *}$ \\ * Unité d'Orthopédie Dento-Faciale, Hôpitaux Universitaires de Strasbourg \\ ** Faculté de Chirurgie-Dentaire de Strasbourg \\ *** Unité de Radiologie Dento-Maxillaire, Hôpitaux Universitaires de Strasbourg \\ **** Unité de Chirurgie buccale, Hôpitaux Universitaires de Strasbourg
}

Les inclusions de canines mandibulaires sont vingt fois moins fréquentes que les maxillaires $(0,35$ à 0,44 \% dans la population générale selon Aydin et al. (2014).

Elles représentent un challenge quotidien aussi bien pour les chirurgiens que pour les orthodontistes lorsqu'une mise en place sur l'arcade est envisagée.

En plus de la décision thérapeutique en lien direct avec ces inclusions et les techniques biomécaniques à mettre en place, les praticiens doivent en même temps gérer les facteurs étiologiques et les éléments associés à ce tableau clinique.

Les cas cliniques présentés dans ce travail viennent illustrer les facteurs causaux et les facteurs associés aux canines mandibulaires retrouvés dans la littérature avec leur prise en charge.

Les causes génétiques sont les plus communément admises notamment par les associations fréquentes entre inclusion, agénésies ou encore certaines malocclusions (Jain et al. 2014). Nous proposons un cas présentant des agénésies des incisives latérales maxillaires 12 et 22 avec une inclusion de la canine mandibulaire 33. Une traction orthodontico-chirurgicale a été réalisée, suivie d'une intervention parodontale ainsi qu'une prise en charge implantaire pour les secteurs des 12 et 22.

Un second cas vient mettre en lumière les associations entre canines incluses et la présence d'une lésion osseuse bénigne de la mandibule. Deux cas, présentant respectivement un kyste dentigère et odontome, illustrent le fait que l'ablation de la lésion suivie d'une traction orthodontique permettent de mettre en place la canine dans la majorité des situations.

Pour finir, un cas d'inclusion idiopathique est montré pour mettre l'accent sur l'importance du bilan clinique et radiologique. Parfois l'extraction de la dent reste la solution de choix.

La mise en place de ces dents peut représenter un véritable challenge thérapeutique à la fois sur plan chirurgical que sur le plan orthodontique. Même si les risques chirurgicaux spécifiques des canines mandibulaires incluses ne sont pas détaillés dans la littérature nous avons à l'esprit les risques liés au geste notamment lorsque des corticotomies doivent être envisagées. De plus, le taux de succès de mise en place de ces éléments dentaires reste relativement faible, d'environ $57 \%$ selon Sainani et al. (2014). Pour le patient le véritable succès clinique ne sera obtenu qu'après avoir résolu tous les éléments de sa malocclusion.

This is an Open Access article distributed under the terms of the Creative Commons Attribution License 4.0, which permits unrestricted use, distribution, and reproduction in any medium, provided the original work is properly cited. 
$63^{\text {ème }}$ Congrès de la SFCO

Nom et adresse du conférencier

Fabien BORNERT

Département de chirurgie buccale, Hôpitaux Universitaires de Strasbourg

Pôle de Médecine et de Chirurgie Bucco-Dentaires

Hôpital Civil CHRU-Strasbourg

67000 Strasbourg (France)

fabien.bornert@chru-strasbourg.fr 